\title{
Uniqueness of the ground state solutions of quasilinear Schrodinger equations
}

Shinji Adachi and Tatsuya Watanabe

\begin{tabular}{|c|l|}
\hline Citation & OCAMI Preprint Series \\
\hline Issue Date & 2009 \\
\hline Type & Preprint \\
\hline Textversion & Author \\
\hline Rights & For personal use only. No other uses without permission. \\
\hline Relation & $\begin{array}{l}\text { The following article has been submitted to Nonlinear Analysis: } \\
\text { Theory, Methods \& Applications. After it is published, it will be } \\
\text { found at https://doi.org/10.1016/j.na.2011.09.015 . }\end{array}$ \\
\hline
\end{tabular}

From: Osaka City University Advanced Mathematical Institute http://www.sci.osaka-cu.ac.jp/OCAMI/publication/preprint/preprint.html 


\title{
Uniqueness of the ground state solutions of quasilinear Schrodinger equations
}

\author{
Shinji Adachi ${ }^{a}$ and Tatsuya Watanabe ${ }^{b}$ \\ ${ }^{a}$ Division of Basic Engineering, Faculty of Engineering, Shizuoka University, \\ 3-5-1 Johoku, Naka-ku, Hamamatsu, 432-8561, Japan \\ ${ }^{b}$ Osaka City University Advanced Mathematical Institute, \\ 3-3-138 Sugimoto, Sumiyoshi-ku, Osaka, 558-8585, Japan
}

\begin{abstract}
We are concerned with the uniqueness result of positive solutions for a class of quasilinear elliptic equation arising from plasma physics. We convert a quasilinear elliptic equation into a semilinear one and show the unique existence of positive radial solution for original equation under the suitable conditions on the power of nonlinearity and quasilinearity. We also investigate non-degeneracy of positive radial solution for converted semilinear elliptic equation.
\end{abstract}

Keywords: quasilinear elliptic equation, uniqueness of positive radial solution, non-degeneracy.

\section{Introduction}

In this paper, we consider the following quasilinear Schrödinger equation:

$$
i \frac{\partial z}{\partial t}=-\Delta z-|z|^{p-1} z-\kappa \Delta\left(|z|^{\alpha}\right)|z|^{\alpha-2} z, \quad(t, x) \in(0, \infty) \times \mathbb{R}^{N}
$$

where $\kappa>0, \alpha>1, N \geq 1$ and $p>1$. Equation (1.1) with $\alpha=2$ derives from a superfluid film equation in plasma physics, which was introduced in $[\mathbf{5}, \mathbf{1 3}]$. We are interested in the standing wave solution of the form: $z(t, x)=u(x) e^{-i \lambda t}, \lambda>0$. Then we obtain the following quasilinear elliptic problem:

$$
-\Delta u+\lambda u-\kappa \Delta\left(|u|^{\alpha}\right)|u|^{\alpha-2} u=|u|^{p-1} u \quad \text { in } \mathbb{R}^{N} .
$$


Equation (1.2) has a variational structure, that is, one can obtain solutions of (1.2) as critical points of the associated functional $J$ defined by

$$
\begin{aligned}
J(u) & =\frac{1}{2} \int_{\mathbb{R}^{N}}|\nabla u|^{2}+\lambda u^{2} d x+\left.\left.\frac{\kappa}{2 \alpha} \int_{\mathbb{R}^{N}}|\nabla| u\right|^{\alpha}\right|^{2} d x-\frac{1}{p+1} \int_{\mathbb{R}^{N}}|u|^{p+1} d x \\
& =\frac{1}{2} \int_{\mathbb{R}^{N}}|\nabla u|^{2}+\lambda u^{2} d x+\frac{\alpha \kappa}{2} \int_{\mathbb{R}^{N}}|\nabla u|^{2}|u|^{2 \alpha-2} d x-\frac{1}{p+1} \int_{\mathbb{R}^{N}}|u|^{p+1} d x .
\end{aligned}
$$

We remark that nonlinear functional $\int_{\mathbb{R}^{N}}|\nabla u|^{2}|u|^{2 \alpha-2} d x$ is not defined on all $H^{1}\left(\mathbb{R}^{N}\right)$ except for $N=1$. Thus the natural function space for $N \geq 2$ is given by

$$
X:=\left\{u \in H^{1}\left(\mathbb{R}^{N}\right) ; \int_{\mathbb{R}^{N}}|\nabla u|^{2}|u|^{2 \alpha-2} d x<\infty\right\} .
$$

Existence of a positive solution of $(1.2)$ has been studied in $[\mathbf{1}, \mathbf{7}, \mathbf{1 4}, \mathbf{1 5}, \mathbf{1 8}]$. The purpose of this paper is to study the uniqueness of the ground state solution of (1.2).

To state the existence of a ground state solution, we use the following notation. We define the ground state energy level and the set of ground states by

$$
\begin{aligned}
& m:=\inf \left\{J(u) ; J^{\prime}(u)=0, u \in X \backslash\{0\}\right\}, \\
& \mathcal{G}:=\left\{w \in X \backslash\{0\} ; J(w)=m, J^{\prime}(w)=0\right\} .
\end{aligned}
$$

In $[\mathbf{1}]$ and [16], they showed the set $\mathcal{G}$ has at least one element which is positive, radially symmetric, decreasing with respect to $r=|x|$ and has the exponential decay. To prove the uniqueness of ground states, we need more precise properties on ground states. Actually we have the following result which was obtained by [8] for the case $\alpha=2$.

Theorem 1.1. Let $\lambda>0, \kappa>0, \alpha>1$ and $1<p<\frac{(2 \alpha-1) N+2}{N-2}$ for $N \geq 3$, $1<p<\infty$ for $N=1,2$. Then $\mathcal{G} \neq \emptyset$ and any $w \in \mathcal{G}$ satisfies the following properties:

(i) $w \in C^{2}\left(\mathbb{R}^{N}, \mathbb{R}\right)$.

(ii) $w(x)>0$ for all $x \in \mathbb{R}^{N}$.

(iii) $w$ is radially symmetric: $w(x)=w(|x|)$ and decreases with respect to $r=|x|$.

(iv) There exist $c, c^{\prime}>0$ such that

$$
\lim _{|x| \rightarrow \infty} e^{\sqrt{\lambda}|x|}(|x|+1)^{\frac{N-1}{2}} w(x)=c, \lim _{r \rightarrow \infty} e^{\sqrt{\lambda} r}(r+1)^{\frac{N-1}{2}} \frac{\partial w}{\partial r}=-c^{\prime} .
$$

When $N=1$, the uniqueness of ground states was already studied in [8]. The main purpose of this paper is to give the uniqueness result for $N \geq 2$. Now Theorem 1.1 implies that

$$
\mathcal{G} \subset\left\{u \in X \cap C^{2} ; u \text { is a positive radial solution of }(1.2)\right\}
$$


We show that the set of positive radial solutions consists of just one element if we assume additional conditions on $\kappa, \lambda, \alpha$ and $p$. Our main result is the following

Theorem 1.2. Assume $N \geq 3, \alpha>1, \alpha-1 \leq p<3 \alpha-3$ and

$$
\max \left\{\frac{\alpha-p}{\alpha(p-1)}, \frac{p}{\alpha(3 \alpha-p-3)}\right\} \leq \kappa \lambda^{\frac{2 \alpha-2}{p-1}}
$$

Then (1.2) has at most one positive radial solution. Hence the ground state solution of (1.2) is unique.

As we will see later, we need a stronger assumption when $N=2$. For detailed conditions on $\kappa, \lambda, \alpha$ and $p$, see Lemma 4.3 below. We obtain the following sufficient conditions for the uniqueness of positive radial solutions in the case $N=2$.

Theorem 1.3. Suppose $N=2, \alpha>2$ and $2 \alpha-1 \leq p<3 \alpha-3$. Then

(i) For every fixed $\kappa>0$, there exists $\lambda_{0}>0$ such that if $\lambda \geq \lambda_{0}$, then (1.2) has at most one positive radial solution.

(ii) For every fixed $\lambda>0$, there exists $\kappa_{0}>0$ such that if $\kappa \geq \kappa_{0}$, then (1.2) has at most one positive radial solution.

To prove Theorem 1.1-1.3, we adapt dual approach as in $[\mathbf{1}, \mathbf{7}]$. More precisely, we convert our quasilinear equation into a semilinear equation by using a suitable translation $f$. We will see that the set of ground states $\mathcal{G}$ has one-to-one correspondence to that of the semilinear problem. This enables us to apply the uniqueness result $[\mathbf{9}, \mathbf{1 2}, \mathbf{1 7}, \mathbf{1 9}]$ for semilinear elliptic equations.

This paper is organized as follows. In Section 2, we introduce the dual approach and prove Theorem 1.1. In Sections 3 and 4, we study the uniqueness for $N \geq 3$ and $N=2$ respectively. Finally in Section 5, we study the non-degeneracy of the ground state solution of the semilinear problem.

\section{Dual approach and Proof of Theorem 1.1}

In this section, we prove Theorem 1.1. Firstly we show the following Pohozaev-type identity.

Lemma 2.1. Let $u \in X$ be a solution of (1.2). Then $u$ satisfies the following identity:

$$
\begin{aligned}
P(u) & :=\frac{N-2}{2 N} \int_{\mathbb{R}^{N}}|\nabla u|^{2}+\kappa \alpha|\nabla u|^{2}|u|^{2 \alpha-2} d x+\frac{\lambda}{2} \int_{\mathbb{R}^{N}} u^{2} d x-\frac{1}{p+1} \int_{\mathbb{R}^{N}}|u|^{p+1} d x \\
& =0 .
\end{aligned}
$$


Proof. For $t>0$, we put $u_{t}(x)=u\left(\frac{x}{t}\right)$. Then we have

$$
\begin{aligned}
& \int_{\mathbb{R}^{N}}\left|\nabla u_{t}\right|^{2} d x=t^{N-2} \int_{\mathbb{R}^{N}}|\nabla u|^{2} d x, \int_{\mathbb{R}^{N}} u_{t}^{2} d x=t^{N} \int_{\mathbb{R}^{N}} u^{2} d x, \\
& \int_{\mathbb{R}^{N}}\left|\nabla u_{t}\right|^{2}\left|u_{t}\right|^{2 \alpha-2} d x=t^{N-2} \int_{\mathbb{R}^{N}}|\nabla u|^{2}|u|^{2 \alpha-2} d x, \int_{\mathbb{R}^{N}}\left|u_{t}\right|^{p+1} d x=t^{N} \int_{\mathbb{R}^{N}}|u|^{p+1} d x .
\end{aligned}
$$

If $u$ is a solution of (1.2), then $\left.\frac{d}{d t} J\left(u_{t}\right)\right|_{t=1}=0$. From this equality, we obtain (2.1).

Next we convert (1.2) into a semilinear problem as in $[\mathbf{1}, \mathbf{7}]$. Let $\tilde{f}$ be a function defined by

$$
\tilde{f}(s):=\int_{0}^{s} \sqrt{1+\kappa \alpha t^{2 \alpha-2}} d t .
$$

Then $\tilde{f}$ is positive, monotone, convex and $C^{\infty}$ on $(0, \infty)$. For $s<0$, we put $\tilde{f}(s)=-\tilde{f}(-s)$. Since $\tilde{f}$ is monotone, we can define the inverse function $f$. Then $f$ satisfies the following ODE:

$$
f^{\prime}(s)=\frac{1}{\sqrt{1+\alpha \kappa f(s)^{2 \alpha-2}}} \quad \text { on } s \in[0, \infty), \quad f(0)=0 .
$$

From (2.2), we can observe that

$$
\begin{aligned}
& f^{\prime \prime}(s)=-\kappa \alpha(\alpha-1) f^{2 \alpha-3}\left(f^{\prime}\right)^{4}=(\alpha-1) \frac{\left(f^{\prime}\right)^{4}}{f}-(\alpha-1) \frac{\left(f^{\prime}\right)^{2}}{f} \\
& f^{\prime \prime \prime}(s)=\frac{1}{f^{2}}\left\{4(\alpha-1)^{2}\left(f^{\prime}\right)^{7}-(6 \alpha-5)(\alpha-1)\left(f^{\prime}\right)^{5}+(\alpha-1)(2 \alpha-1)\left(f^{\prime}\right)^{3}\right\} .
\end{aligned}
$$

The next two lemmas play important roles in Sections 3-5.

Lemma 2.2. $f(s)$ satisfies the following properties:

(i) $f(s) \leq s, f^{\prime}(s) \in(0,1], f^{\prime \prime}(s) \leq 0$ for all $s \geq 0$.

(ii) $\frac{1}{\alpha} f(s) \leq s f^{\prime}(s) \leq f(s)$ for all $s \geq 0$.

(iii) $\left(\frac{f(s)}{s f^{\prime}(s)}\right)^{\prime}>0$ for all $s>0$.

\section{Lemma 2.3. It follows}

(i) $\lim _{s \rightarrow \infty} \frac{f(s)}{s^{\frac{1}{\alpha}}}=\left(\frac{\alpha}{\kappa}\right)^{\frac{1}{2 \alpha}}, \lim _{s \rightarrow 0} \frac{f(s)}{s}=1$.

(ii) $\lim _{s \rightarrow \infty} \frac{f^{\prime}(s)}{s^{\frac{1-\alpha}{\alpha}}}=\frac{1}{\alpha}\left(\frac{\alpha}{\kappa}\right)^{\frac{1}{2 \alpha}}$.

(iii) $\lim _{s \rightarrow \infty} \frac{f(s)}{s f^{\prime}(s)}=\alpha$.

For the proof of (i) and (ii) of Lemma 2.2 and Lemma 2.3, we refer to [1]. Here we give the proof of (iii) of Lemma 2.2. 
Proof. By (2.3) and (ii) of Lemma 2.2, we have

$$
\begin{aligned}
\left(\frac{f}{s f^{\prime}}\right)^{\prime} & =\frac{1}{\left(s f^{\prime}\right)^{2}}\left(s\left(f^{\prime}\right)^{2}-f f^{\prime}-s f f^{\prime \prime}\right) \\
& \geq \frac{1}{\left(s f^{\prime}\right)^{2}}\left(s\left(f^{\prime}\right)^{2}-s\left(f^{\prime}\right)^{2}-s f f^{\prime \prime}\right) \\
& =\frac{-f f^{\prime \prime}}{s\left(f^{\prime}\right)^{2}}=(\alpha-1) \frac{1-\left(f^{\prime}\right)^{2}}{s} .
\end{aligned}
$$

Since $0<f^{\prime}<1$ for all $s>0$, we obtain the desired inequality.

Using the function $f$, we consider the following semilinear problem:

$$
-\Delta v+\lambda f(v) f^{\prime}(v)=|f(v)|^{p-1} f(v) f^{\prime}(v) \text { in } \mathbb{R}^{N} .
$$

The functional associated to (2.5) is defined by

$$
I(v)=\frac{1}{2} \int_{\mathbb{R}^{N}}|\nabla v|^{2}+\lambda f(v)^{2} d x-\frac{1}{p+1} \int_{\mathbb{R}^{N}}|f(v)|^{p+1} d x .
$$

From Lemma 2.3, we can see that $I$ is well-defined on $H^{1}\left(\mathbb{R}^{N}\right)$ (see $[\mathbf{1}, \mathbf{7}]$ for the proof). Moreover we have the following relation between (1.2) and (2.5), which was already shown in $[\mathbf{1}, \mathbf{7}]$. For the sake of completeness, we give the proof.

Lemma 2.4. Suppose $v$ is a nontrivial critical point of $I$ and $v>0$. Then $u=f(v)$ is a positive solution of (1.2).

Proof. We can easily see that if $v \in H^{1}\left(\mathbb{R}^{N}\right)$ is a nontrivial critical point of $I(v)$, then $v$ is a solution of (2.5). By standard elliptic regularity theory, we see that $v \in C^{2}\left(\mathbb{R}^{N}\right)$. Moreover $v>0$ implies $u>0$. Since $f \in C^{\infty}(0, \infty)$, we also have $u \in C^{2}\left(\mathbb{R}^{N}\right)$.

For $v=\tilde{f}(u)$, we have

$$
\nabla v=\tilde{f}^{\prime}(u) \nabla u, \quad \Delta v=\tilde{f}^{\prime \prime}(u)|\nabla u|^{2}+\tilde{f}^{\prime}(u) \Delta u .
$$

From (2.2), it follows

$$
\begin{aligned}
& \tilde{f}^{\prime}(s)=\frac{1}{f^{\prime}(\tilde{f}(s))}=\sqrt{1+\kappa \alpha|f(\tilde{f}(s))|^{2 \alpha-2}}=\sqrt{1+\kappa \alpha|s|^{2 \alpha-2}}, \\
& \tilde{f}^{\prime \prime}(s)=\frac{\kappa \alpha(\alpha-1)|s|^{2 \alpha-4} s}{\sqrt{1+\kappa \alpha|s|^{2 \alpha-2}}} .
\end{aligned}
$$

Thus we have

$$
\Delta v=\frac{\kappa \alpha(\alpha-1)|u|^{2 \alpha-4} u}{\sqrt{1+\kappa \alpha|u|^{2 \alpha-2}}}|\nabla u|^{2}+\sqrt{1+\kappa \alpha|u|^{2 \alpha-2}} \Delta u
$$


From (2.5), we can observe that $u$ satisfies

$$
-\Delta u-\kappa \alpha|u|^{2 \alpha-2} \Delta u-\kappa \alpha(\alpha-1)|u|^{2 \alpha-4} u|\nabla u|^{2}+\lambda u=|u|^{p-1} u .
$$

Now $u>0$ implies $|u|^{\alpha} \in C^{2}$. Then it follows from

$$
\begin{aligned}
\Delta\left(|u|^{\alpha}\right) & =\operatorname{div}\left(\alpha|u|^{\alpha-2} u \nabla u\right) \\
& =\alpha|u|^{\alpha-2} u \Delta u+\nabla u \cdot \nabla\left(\alpha|u|^{\alpha-2} u\right) \\
& =\alpha|u|^{\alpha-2} u \Delta u+\alpha(\alpha-1)|u|^{\alpha-2}|\nabla u|^{2}
\end{aligned}
$$

that

$$
\Delta\left(|u|^{\alpha}\right)|u|^{\alpha-2} u=\alpha|u|^{2 \alpha-2} \Delta u+\alpha(\alpha-1)|u|^{2 \alpha-4} u|\nabla u|^{2} .
$$

Thus from (2.6) and (2.7), we see that if $v$ is a nontrivial critical point of $I$ and $v>0$, then $u=f(v)$ is a positive solution of (1.2).

Remark 2.5. If $\alpha \geq 2$, then $f \in C^{2}[0, \infty)$ and $|f(v)|^{\alpha} \in C^{2}$ for any nontrivial critical point $v$ of $I$. Thus Lemma 2.4 holds for any nontrivial critical point (possibly signchanging) of $I$ if $\alpha \geq 2$.

Lemma 2.4 tells us that we have only to show the existence of a positive solution of (2.5) in order to find a positive solution of (1.2). However to show the existence of a ground state solution, we need more informations on the relation between (1.2) and (2.5). Actually we have the following relations.

\section{Lemma 2.6.}

(i) It follows $X=f\left(H^{1}\left(\mathbb{R}^{N}\right)\right)$, that is, $X=\left\{f(v) ; v \in H^{1}\left(\mathbb{R}^{N}\right)\right\}=: Y$.

(ii) For any $v \in H^{1}\left(\mathbb{R}^{N}\right)$, we put $u=f(v)$. Then it follows $J(u)=I(v)$.

Proof. (i) First we show $Y \subset X$. For $v \in H^{1}\left(\mathbb{R}^{N}\right)$, we put $u=f(v)$. Then we have

$$
|\nabla f(v)|^{2}=\left|f^{\prime}(v)\right|^{2}|\nabla v|^{2}=\frac{1}{1+\kappa \alpha|f(v)|^{2 \alpha-2}}|\nabla v|^{2}
$$

By (i) of Lemma 2.2 and (2.2), we obtain

$$
\int_{\mathbb{R}^{N}}|\nabla u|^{2}+u^{2} d x+\kappa \alpha \int_{\mathbb{R}^{N}}|\nabla u|^{2}|u|^{2 \alpha-2} d x=\int_{\mathbb{R}^{N}}|\nabla v|^{2}+f(v)^{2} d x \leq C\|v\|_{H^{1}}^{2}<\infty .
$$

Thus it follows $Y \subset X$. 
To show $X \subset Y$, it suffices to show $\tilde{f}(u) \in H^{1}\left(\mathbb{R}^{N}\right)$ for all $u \in X$. For $u \in X$, we put $v=\tilde{f}(u)$. Then it follows

$$
\int_{\mathbb{R}^{N}}|\nabla v|^{2} d x=\int_{\mathbb{R}^{N}}\left|(\tilde{f})^{\prime}(u)\right|^{2}|\nabla u|^{2} d x=\int_{\mathbb{R}^{N}}\left(1+\kappa \alpha|u|^{2 \alpha-2}\right)|\nabla u|^{2} d x<\infty .
$$

Next by (i) of Lemma 2.3, it follows

$$
\lim _{s \rightarrow 0} \frac{\tilde{f}(s)}{s}=1, \quad \lim _{s \rightarrow \infty} \frac{\tilde{f}(s)}{s^{\alpha}}=c
$$

for some $c>0$. Thus there exist constants $C_{1}, C_{2}>0$ such that

$$
|\tilde{f}(s)| \leq C_{1} \chi_{|s| \leq 1}|s|+C_{2} \chi_{|s| \geq 1}|s|^{\alpha} \quad \text { for all } s \in \mathbb{R}
$$

Then we have

$$
|v|^{2} \leq C_{1} \chi_{|u| \leq 1}|u|^{2}+C_{2} \chi_{|u| \geq 1}|u|^{2 \alpha} \leq C_{1}|u|^{2}+C_{2}|u|^{\frac{2 N \alpha}{N-2}}
$$

By Sobolev's inequality, we obtain

$$
\begin{aligned}
\int_{\mathbb{R}^{N}}|v|^{2} d x & \leq C_{1} \int_{\mathbb{R}^{N}}|u|^{2} d x+C_{2} \int_{\mathbb{R}^{N}}|u|^{\frac{2 N \alpha}{N-2}} d x \\
& \leq C_{1} \int_{\mathbb{R}^{N}}|u|^{2} d x+C_{2}^{\prime}\left(\int_{\mathbb{R}^{N}} \alpha^{2}|\nabla u|^{2}|u|^{2 \alpha-2} d x\right)^{\frac{N}{N-2}}<\infty .
\end{aligned}
$$

Thus it follows $X \subset Y$ and hence $X=Y$.

(ii) We substitute $u=f(v)$ into $J(u)$. Then from (2.2), it follows

$$
\begin{aligned}
J(u)= & \frac{1}{2} \int_{\mathbb{R}^{N}}|\nabla f(v)|^{2}+\lambda f(v)^{2} d x+\frac{\kappa \alpha}{2} \int_{\mathbb{R}^{N}}|\nabla f(v)|^{2}|f(v)|^{2 \alpha-2} d x \\
& \quad-\frac{1}{p+1} \int_{\mathbb{R}_{N}}|f(v)|^{p+1} d x \\
= & \frac{1}{2} \int_{\mathbb{R}^{N}}|\nabla v|^{2}\left(1+\kappa \alpha|f(v)|^{2 \alpha-2}\right)\left|f^{\prime}(v)\right|^{2}+\lambda f(v)^{2} d x-\frac{1}{p+1} \int_{\mathbb{R}^{N}}|f(v)|^{p+1} d x \\
= & \frac{1}{2} \int_{\mathbb{R}^{N}}|\nabla v|^{2}+\lambda f(v)^{2} d x-\frac{1}{p+1} \int_{\mathbb{R}^{N}}|f(v)|^{p+1} d x \\
= & I(v) .
\end{aligned}
$$

and we obtain (ii).

Now we are ready to prove Theorem 1.1. 
Proof of Theorem 1.1. We argue as in [8]. By the results due to $[\mathbf{3}, \mathbf{4}, \mathbf{1 1}]$, there exists $\tilde{w} \in H^{1}\left(\mathbb{R}^{N}\right)$ such that $\tilde{w}>0$, radial and

$$
I(\tilde{w})=\inf \left\{I(v) ; I^{\prime}(v)=0, v \in H^{1}\left(\mathbb{R}^{N}\right) \backslash\{0\}\right\}
$$

By Lemma 2.4, $w=f(\tilde{w})$ satisfies $J^{\prime}(w)=0$. We claim that $w \in \mathcal{G}$.

Indeed we define

$$
\tilde{P}(v):=\frac{N-2}{2 N} \int_{\mathbb{R}^{N}}|\nabla v|^{2} d x+\frac{\lambda}{2} \int_{\mathbb{R}^{N}} f(v)^{2} d x-\frac{1}{p+1} \int_{\mathbb{R}^{N}}|f(v)|^{p+1} d x, v \in H^{1}\left(\mathbb{R}^{N}\right) .
$$

Then it follows $P(u)=\tilde{P}(v)$ for $u=f(v)$ where $P(u)$ was defined in (2.1). Moreover $\tilde{w}$ can be characterized by

$$
\tilde{w} \in A:=\left\{v \in H^{1}\left(\mathbb{R}^{N}\right) ; \tilde{P}(v)=0\right\}, \quad I(\tilde{w})=\inf _{v \in A} I(v) .
$$

Now let $u \in X$ be a nontrivial critical point of $J$. Then from (2.1), we have

$$
\begin{aligned}
J(u) & =\frac{1}{N} \int_{\mathbb{R}^{N}}|\nabla u|^{2}+\alpha \kappa|\nabla u|^{2}|u|^{2 \alpha-2} d x \\
& =\frac{1}{N} \int_{\mathbb{R}^{N}}|\nabla u|^{2} d x+\left.\left.\frac{\kappa}{\alpha N} \int_{\mathbb{R}^{N}}|\nabla| u\right|^{\alpha}\right|^{2} d x .
\end{aligned}
$$

By the pointwise inequality $|\nabla| u(x)|| \leq|\nabla u(x)|$ a.e. $x \in \mathbb{R}^{N}$, it follows

$$
J(u) \geq J(|u|)=I(\tilde{f}(|u|)) .
$$

If $N=2$, then $(2.1)$ implies $P(|u|)=0$ and hence $\tilde{P}(\tilde{f}(|u|))=0$. Then we obtain

$$
J(u) \geq I(\tilde{f}(|u|)) \geq I(\tilde{w})=J(w) .
$$

If $N \geq 3$, we distinguish cases $P(|u|)=0$ and $P(|u|)<0$. If $P(|u|)=0$, then we have $J(u) \geq J(w)$ as in the case $N=2$. Suppose $P(|u|)=\tilde{P}(\tilde{f}(|u|))<0$. We put $\tilde{v}=\tilde{f}(|u|)$ and define $\tilde{v}_{\theta}(x)=\tilde{v}\left(\frac{x}{\theta}\right)$ for $\theta \in(0,1)$. We can see that there exists $\theta_{0} \in(0,1)$ such that $\tilde{P}\left(\tilde{v}_{\theta_{0}}\right)=0$, that is, $\tilde{v}_{\theta_{0}} \in A$. Then we have

$$
\begin{aligned}
I\left(\tilde{v}_{\theta_{0}}\right) & =\frac{\theta_{0}^{N-2}}{N} \int_{\mathbb{R}^{N}}|\nabla \tilde{v}|^{2} d x \\
& =\left.\frac{\theta_{0}^{N-2}}{N} \int_{\mathbb{R}^{N}}|\nabla| u\right|^{2}+\left.\left.\frac{\kappa}{\alpha}|\nabla| u\right|^{\alpha}\right|^{2} d x \\
& \leq \frac{\theta_{0}^{N-2}}{N} \int_{\mathbb{R}^{N}}|\nabla u|^{2}+\left.\left.\frac{\kappa}{\alpha}|\nabla| u\right|^{\alpha}\right|^{2} d x \\
& =\theta_{0}^{N-2} J(u)<J(u) .
\end{aligned}
$$


Since $\tilde{v}_{\theta_{0}} \in A$, we obtain

$$
J(u)>I\left(\tilde{v}_{\theta_{0}}\right) \geq I(\tilde{w})=J(w) .
$$

This implies $w \in \mathcal{G}$.

Next we show that if $w \in \mathcal{G}$, then $|w| \in \mathcal{G}$. If $P(|w|)<0$, then we get

$$
J(w)>I\left(\tilde{v}_{\theta_{0}}\right)=J(w) .
$$

This is a contradiction. Thus it follows $P(|w|)=0$. Arguing as above, we obtain $J(w)=$ $J(|w|)$. This implies $|w| \in \mathcal{G}$.

Next we show properties (i)-(iv).

(i) We can see if $w \in \mathcal{G}$, then $w \in L_{l o c}^{\infty}\left(\mathbb{R}^{N}\right)$. By the elliptic regularity theory, it follows $w \in C^{2}\left(\mathbb{R}^{N}\right)$.

(ii) Since $|w| \in \mathcal{G}$ for any $w \in \mathcal{G}$, it follows $w \geq 0$. We put $\tilde{w}=\tilde{f}(w)$. By the maximum principle, we have $\tilde{w}>0$. This implies $w>0$.

(iii) We observe that if $w \in \mathcal{G}$, then $\tilde{w}=\tilde{f}(w)$ is a ground state solution of (2.5). In fact for any nontrivial critical point $u$ of $J, v=\tilde{f}(u)$ is a nontrivial critical point of $I$. Then by Lemma 2.6, we have

$$
I(v)=J(u) \geq m=J(w)=I(\tilde{w}) .
$$

This implies $\tilde{w}$ is a ground state solution of (2.5).

By the result of $[6]$, any ground state solution of (2.5) is radially symmetric and decreasing with respect to $r=|x|$. We can easily see that $w(x)=w(|x|)$ if and only if $\tilde{w}(x)=\tilde{f}(w(x))$ satisfies $\tilde{w}(x)=\tilde{w}(|x|)$. Thus claim (iii) holds.

(iv) Let $w \in \mathcal{G}$ and $\tilde{w}=\tilde{f}(w)$. From (ii), $\tilde{w}$ is a positive radial solution of (2.5). Then by the standard comparison principle, it follows

$$
\left|D^{k} \tilde{w}(x)\right| \leq c e^{-\delta|x|} \quad \text { for all } \delta \in(0, \sqrt{\lambda}), x \in \mathbb{R}^{N},|k| \leq 2 \text { and some } c>0 .
$$

By Lemma 2.2, we can see that

$$
\left|D^{k} w(x)\right| \leq c e^{-\delta|x|} \quad \text { for all } \delta \in(0, \sqrt{\lambda}), x \in \mathbb{R}^{N},|k| \leq 2 \text { and some } c>0 .
$$

Thus for $|x| \gg 1$, we have

$$
-\Delta w+\lambda w \leq c e^{-p \delta|x|}+c e^{-(2 \alpha-1) \delta|x|} .
$$

Since $2 \alpha-1>1$ and we can take $\delta$ arbitrarily close to $\sqrt{\lambda}$, it follows

$$
-\Delta w+\lambda w=o(G(x)) \text { as }|x| \rightarrow \infty,
$$

where $G$ is the fundamental solution of $-\Delta+\lambda I$. Then by Gidas-Ni-Nirenberg's asymptotic result $[\mathbf{1 0}]$, we obtain (iv).

Next we give a relation on the sets of positive radial solutions of (1.2) and (2.5). 
Lemma 2.7. It follows

$$
\begin{aligned}
& \left\{u \in X \cap C^{2}\left(\mathbb{R}^{N}\right) ; J^{\prime}(u)=0, u>0, u(x)=u(|x|)\right\} \\
& \quad=f\left(\left\{v \in H^{1} \cap C^{2}\left(\mathbb{R}^{N}\right) ; I^{\prime}(v)=0, v>0, v(x)=v(|x|)\right\}\right) .
\end{aligned}
$$

Proof. By Lemma 2.4, we know that

$$
\left\{u \in X \cap C^{2}\left(\mathbb{R}^{N}\right) ; J^{\prime}(u)=0, u>0\right\} \supseteq\left\{f(v) ; I^{\prime}(v)=0, v>0, v \in H^{1} \cap C^{2}\left(\mathbb{R}^{N}\right)\right\} .
$$

Suppose the equality does not hold. Then there exists $u_{0} \in X$ such that $u_{0}$ is a positive solution of (1.2) but $u_{0} \neq f(v)$ for any positive solution $v \in H^{1}\left(\mathbb{R}^{N}\right)$ of $(2.5)$.

On the other hand by (i) of Lemma 2.6, we know if $u_{0} \in X$, then there exists $v_{0}$ such that $u_{0}=f\left(v_{0}\right)$. Since $u_{0}$ is a positive solution of $(1.2)$, we can see that $v_{0}$ is a positive solution of (2.5). This is a contradiction. Thus we have

$$
\left\{u \in X \cap C^{2}\left(\mathbb{R}^{N}\right) ; J^{\prime}(u)=0, u>0\right\}=\left\{f(v) ; I^{\prime}(v)=0, v>0, v \in H^{1} \cap C^{2}\left(\mathbb{R}^{N}\right)\right\} .
$$

Finally we can easily see that $u(x)=u(|x|)$ if and only if $v(x)=\tilde{f}(u(x))$ satisfies $v(x)=$ $v(|x|)$.

Lemma 2.7 tells us that if (2.5) has at most one positive radial solution $v$, then $(1.2)$ also has at most one positive radial solution $u=f(v)$. Thus we have only to study the uniqueness of the positive solution of semilinear problem (2.5).

Finally in this section, we give the non-existence result for $p \geq \frac{(2 \alpha-1) N+2}{N-2}$, which is an easy consequence of the Pohozaev identity.

Theorem 2.8. Suppose $p \geq \frac{(2 \alpha-1) N+2}{N-2}$. Then (1.2) has no nontrivial solution $u \in X$.

Proof. Suppose $u \in X$ is a nontrivial solution of (1.2) and $p \geq \frac{(2 \alpha-1) N+2}{N-2}$. From $J^{\prime}(u) u=0$, we have

$$
\int_{\mathbb{R}^{N}}|\nabla u|^{2}+\lambda u^{2}+\kappa \alpha^{2}|\nabla u|^{2}|u|^{2 \alpha-2} d x-\int_{\mathbb{R}^{N}}|u|^{p+1} d x=0 .
$$

On the other hand, $u$ satisfies (2.1). Then we obtain

$$
\begin{aligned}
\frac{(\alpha-1)(N-2)}{2 \alpha} \int_{\mathbb{R}^{N}}|\nabla u|^{2} d x & +\frac{(\alpha-1) N+2}{2 \alpha} \int_{\mathbb{R}^{N}} \lambda u^{2} d x \\
& =\left(\frac{N}{p+1}-\frac{N-2}{2 \alpha}\right) \int_{\mathbb{R}^{N}}|u|^{p+1} d x .
\end{aligned}
$$


Since the left hand side is positive, it follows

$$
\frac{N}{p+1}-\frac{N-2}{2 \alpha}>0, \text { that is, } p<\frac{(2 \alpha-1) N+2}{N-2} \text {. }
$$

This is a contradiction.

\section{Uniqueness of positive radial solutions for $N \geq 3$}

In this section, we study the uniqueness of the positive radial solution of (2.5). We put

$$
g(s)=f(s)^{p} f^{\prime}(s)-\lambda f(s) f^{\prime}(s) \text { for } s \geq 0 \text { and } K_{g}(s):=\frac{s g^{\prime}(s)}{g(s)} .
$$

We apply the following uniqueness result due to Serrin and Tang [19].

Proposition 3.1 [19]. Suppose that there exists $b>0$ such that

(i) $g$ is continuous on $(0, \infty), g(s) \leq 0$ on $(0, b]$ and $g(s)>0$ for $s>b$.

(ii) $g \in C^{1}(b, \infty)$ and $K_{g}^{\prime}(s) \leq 0$ on $(b, \infty)$.

Then the semilinear problem:

$$
-\Delta u=g(u) \text { in } \mathbb{R}^{N}, u>0, u \rightarrow 0 \text { as }|x| \rightarrow \infty, u(0)=\max u(x)
$$

has at most one positive radial solution.

Now we can see that $g$ defined in (3.1) is of the class $C^{1}[0, \infty)$ and

$$
g(s)=0 \Longleftrightarrow f^{p-1}(s)=\lambda \Longleftrightarrow s=f^{-1}\left(\lambda^{\frac{1}{p-1}}\right) .
$$

We put $b:=f^{-1}\left(\lambda^{\frac{1}{p-1}}\right)$. Since $(s-b) g(s)=(s-b) f f^{\prime}\left(f^{p-1}-\lambda\right)$, we can see (i) of Proposition 3.1 holds.

Lemma 3.2. Assume $\alpha-1 \leq p<3 \alpha-3$ and

$$
\max \left\{\frac{\alpha-p}{\alpha(p-1)}, \frac{p}{\alpha(3 \alpha-p-3)}\right\} \leq \kappa \lambda^{\frac{2 \alpha-2}{p-1}} .
$$

Then $g$ satisfies (ii) of Proposition 3.1.

Proof. We observe that

$$
K_{g}^{\prime}(s)=\frac{1}{g(s)^{2}}\left(g^{\prime \prime}(s) g(s) s+g^{\prime}(s) g(s)-\left(g^{\prime}(s)\right)^{2} s\right)
$$


Thus we have only to show that $s g^{\prime \prime} g+g^{\prime} g-s\left(g^{\prime}\right)^{2}<0$ for $s>b$.

Firstly we compute $g^{\prime}$ and $g^{\prime \prime}$. It follows

$$
\begin{aligned}
g(s)= & f^{p} f^{\prime}-\lambda f f^{\prime}=f f^{\prime}\left(f^{p-1}-\lambda\right), \\
g^{\prime}(s)= & (p-1) f^{p-1}\left(f^{\prime}\right)^{2}+\left(f^{\prime}\right)^{2}\left(f^{p-1}-\lambda\right)+f f^{\prime \prime}\left(f^{p-1}-\lambda\right), \\
g^{\prime \prime}(s)= & (p-1)^{2} f^{p-2}\left(f^{\prime}\right)^{3}+2(p-1) f^{p-1} f^{\prime} f^{\prime \prime}+2 f^{\prime} f^{\prime \prime}\left(f^{p-1}-\lambda\right)+(p-1) f^{p-2}\left(f^{\prime}\right)^{3} \\
& \quad+f^{\prime} f^{\prime \prime}\left(f^{p-1}-\lambda\right)+f f^{\prime \prime \prime}\left(f^{p-1}-\lambda\right)+(p-1) f^{p-1} f^{\prime} f^{\prime \prime} \\
& =p(p-1) f^{p-2}\left(f^{\prime}\right)^{3}+3(p-1) f^{p-1} f^{\prime} f^{\prime \prime}+3 f^{\prime} f^{\prime \prime}\left(f^{p-1}-\lambda\right)+f f^{\prime \prime \prime}\left(f^{p-1}-\lambda\right) .
\end{aligned}
$$

Then we have

$$
\begin{aligned}
& s g^{\prime \prime} g+g^{\prime} g-s\left(g^{\prime}\right)^{2} \\
& =\left(f^{p-1}-\lambda\right)^{2}\left(s f^{2} f^{\prime} f^{\prime \prime \prime}+s f\left(f^{\prime}\right)^{2} f^{\prime \prime}-s\left(f^{\prime}\right)^{4}-s f^{2}\left(f^{\prime \prime}\right)^{2}+f\left(f^{\prime}\right)^{3}+f^{2} f^{\prime} f^{\prime \prime}\right) \\
& \quad+\left(f^{p-1}-\lambda\right)(p-1) f^{p-1}\left(f^{\prime}\right)^{2}\left((p-2) s\left(f^{\prime}\right)^{2}+s f f^{\prime \prime}+f f^{\prime}\right) \\
& \quad-(p-1)^{2} s f^{2 p-2}\left(f^{\prime}\right)^{4} .
\end{aligned}
$$

Next we express $s g^{\prime \prime} g+g^{\prime} g-s\left(g^{\prime}\right)^{2}$ in terms of $f$ and $f^{\prime}$ and regard as a polynomial of $f^{p-1}-\lambda$. From (2.3) and (2.4), we have

$$
\begin{aligned}
& s g^{\prime \prime} g+g^{\prime} g-s\left(g^{\prime}\right)^{2} \\
& =\left(f^{p-1}-\lambda\right)^{2}\left((\alpha-1) f f^{\prime 5}-4(\alpha-1)^{2} s f^{\prime 6}+3(\alpha-1)^{2} s f^{\prime 8}+(\alpha-2) f^{\prime 3}\left(\alpha s f^{\prime}-f\right)\right) \\
& \quad+(p-1) f^{p-1}\left(f^{p-1}-\lambda\right)\left((p-\alpha-1) s f^{\prime 4}+(\alpha-1) s f^{\prime 6}+f f^{\prime 3}\right) \\
& \quad-(p-1)^{2} s f^{2 p-2} f^{\prime 4} .
\end{aligned}
$$

Now we use

$$
\begin{aligned}
& \left(f^{p-1}-\lambda\right) f^{p-1}=\left(f^{p-1}-\lambda\right)^{2}+\lambda f^{p-1}-\lambda^{2}=\left(f^{p-1}-\lambda\right)^{2}+\lambda\left(f^{p-1}-\lambda\right), \\
& f^{2 p-2}=\left(f^{p-1}-\lambda\right)^{2}+2 \lambda f^{p-1}-\lambda^{2}=\left(f^{p-1}-\lambda\right)^{2}+2 \lambda\left(f^{p-1}-\lambda\right)+\lambda^{2} .
\end{aligned}
$$

Thus we obtain

$$
\begin{aligned}
& s g^{\prime \prime} g+g^{\prime} g-s\left(g^{\prime}\right)^{2} \\
&=\left(f^{p-1}-\lambda\right)^{2}\left((\alpha-1) f f^{\prime 5}-4(\alpha-1)^{2} s f^{\prime 6}+3(\alpha-1)^{2} s f^{\prime 8}+(\alpha-2) f^{\prime 3}\left(\alpha s f^{\prime}-f\right)\right) \\
&+(p-1)\left(\left(f^{p-1}-\lambda\right)^{2}+\lambda\left(f^{p-1}-\lambda\right)\right)\left((p-\alpha-1) s f^{\prime 4}+(\alpha-1) s f^{\prime 6}+f f^{\prime 3}\right) \\
&-(p-1)^{2}\left(\left(f^{p-1}-\lambda\right)^{2}+2 \lambda\left(f^{p-1}-\lambda\right)+\lambda^{2}\right) s f^{\prime 4} \\
&=\left(f^{p-1}-\lambda\right)^{2}\left((\alpha-1) f f^{\prime 5}-4(\alpha-1)^{2} s f^{\prime 6}+3(\alpha-1) s f^{\prime 8}+(\alpha-2) f^{\prime 3}\left(\alpha s f^{\prime}-f\right)\right. \\
&\left.\quad+(p-1)(p-\alpha-1) s f^{\prime 4}+(p-1)(\alpha-1) s f^{\prime 6}+(p-1) f f^{\prime 3}-(p-1)^{2} s f^{\prime 4}\right)
\end{aligned}
$$




$$
\begin{aligned}
& +\lambda(p-1)\left(f^{p-1}-\lambda\right)\left((p-\alpha-1) s f^{4}+(\alpha-1) s f^{\prime 6}+f f^{\prime 3}-2(p-1) s f^{\prime 4}\right) \\
& -\lambda^{2}(p-1)^{2} s f^{\prime 4} \\
= & \left(f^{p-1}-\lambda\right)^{2}\left((\alpha-1) f^{\prime 5}\left(f-(4 \alpha-p-3) s f^{\prime}+3(\alpha-1) s f^{\prime 3}\right)-(p-\alpha+1) f^{\prime 3}\left(\alpha s f^{\prime}-f\right)\right) \\
& +\lambda(p-1)\left(f^{p-1}-\lambda\right)\left(-(\alpha+p-1) s f^{\prime 4}+f f^{\prime 3}+(\alpha-1) s f^{\prime 6}\right) \\
& -\lambda^{2}(p-1)^{2} s f^{\prime 4} \\
= & :\left(f^{p-1}-\lambda\right)^{2} H_{1}(s)+\lambda(p-1)\left(f^{p-1}-\lambda\right) H_{2}(s)-\lambda^{2}(p-1)^{2} s f^{\prime 4} .
\end{aligned}
$$

First we study the sign of $H_{2}(s)$. It follows

$$
H_{2}(s)=f^{\prime 2}\left\{-f^{\prime}\left(\alpha s f^{\prime}-f\right)-\left((p-1)-(\alpha-1) f^{\prime 2}\right) s f^{\prime 2}\right\}
$$

From Lemma 2.2, we know that $\alpha s f^{\prime}-f \geq 0$ for all $s \geq 0$. Moreover from the fact that $f^{\prime}$ is decreasing, we have

$$
(p-1)-(\alpha-1) f^{\prime 2} \geq p-1-(\alpha-1) f^{\prime}(b)^{2}=\frac{p-\alpha+\kappa \alpha(p-1) \lambda^{\frac{2 \alpha-2}{p-1}}}{1+\alpha \kappa \lambda^{\frac{2 \alpha-2}{p-1}}} .
$$

From (3.2), it follows $H_{2}(s) \leq 0$ for $s>b$.

Next we investigate the sign of $H_{1}(s)$. We have

$$
\begin{aligned}
H_{1}(s) & =(\alpha-1) f^{\prime 5}\left(f-(4 \alpha-p-3) s f^{\prime}+3(\alpha-1) s f^{\prime 3}\right)-(p-\alpha+1) f^{\prime 3}\left(\alpha s f^{\prime}-f\right) \\
& =:(\alpha-1) f^{\prime 5} H_{3}(s)-(p-\alpha+1) f^{\prime 3}\left(\alpha s f^{\prime}-f\right) .
\end{aligned}
$$

Since $p-\alpha+1 \geq 0$, it suffices to show that $H_{3}(s) \leq 0$ in order to prove $H_{1}(s) \leq 0$.

Now we observe that

$$
H_{3}(s)=-\left(\alpha s f^{\prime}-f\right)-s f^{\prime}\left((3 \alpha-p-3)-3(\alpha-1) f^{\prime 2}\right) .
$$

From $p<3 \alpha-3$ and the fact that $f^{\prime}$ is decreasing, we have

$$
\begin{aligned}
(3 \alpha-p-3)-3(\alpha-1) f^{\prime}(s)^{2} & \geq(3 \alpha-p-3)-3(\alpha-1) f^{\prime}(b)^{2} \\
& =(3 \alpha-p-3)-\frac{3(\alpha-1)}{1+\alpha \lambda f(b)^{2 \alpha-2}} \\
& =\frac{-p+\alpha(3 \alpha-p-3) \kappa \lambda^{\frac{2 \alpha-2}{p-1}}}{1+\alpha \kappa \lambda^{\frac{2 \alpha-2}{p-1}}} .
\end{aligned}
$$

We see that

$$
-p+\alpha(3 \alpha-p-3) \kappa \lambda^{\frac{2 \alpha-2}{p-1}} \geq 0 \Longleftrightarrow \frac{p}{\alpha(3 \alpha-p-3)} \leq \kappa \lambda^{\frac{2 \alpha-2}{p-1}}
$$


Thus we have $H_{3}(s) \leq 0$ and hence $H_{1}(s) \leq 0$. From $(3.3)$, we obtain $K_{g}^{\prime}(s)<0$ for $s>b$.

By Lemma 3.2, we can apply Proposition 3.1. Hence we obtain the uniqueness of positive radial solutions of (1.2) when $N \geq 3$ and the proof of Theorem 1.2 is complete.

\section{Uniqueness of positive radial solutions for $N=2$}

In this section, we study the uniqueness of positive radial solutions of (2.5) for $N=2$. Under same notation as in Section 3, we apply the following uniqueness result due to Mcleod and Serrin [17].

Proposition 4.1 [17]. Suppose that there exist $b>0$ and $\tau \geq 1$ such that

(i) $g \in C^{1}[0, \infty), g(0)=0, g^{\prime}(0)<0$.

(ii) $g(s)<0$ for $s \in(0, b), g(s)>0$ for $s \in(b, \infty)$.

(iii) $g^{\prime}(b)>0$.

(iv) $\left(\frac{g(s)}{s^{\tau}}\right)^{\prime}>0$ for $s>0, s \neq b$.

(v) $\left(s\left(\frac{g(s)}{s^{\tau}}\right)^{\prime}\right)^{\prime}<0$ for $s>b$.

Then the semilinear problem:

$$
-\Delta u=g(u) \text { in } \mathbb{R}^{2}, u>0, u \rightarrow 0 \text { as }|x| \rightarrow \infty, u(0)=\max u(x)
$$

has at most one positive radial solution.

As mentioned in [12], (iv) and (v) of Proposition 4.1 imply (ii) of Proposition 3.1. This means that we need a stronger condition on the nonlinearity to show the uniqueness for $N=2$. Thus we have to restrict $\lambda, \kappa, \alpha$ and $p$ more strongly to show (iv) and (v).

Now we can see $g$ defined in (3.1) satisfies (i)-(iii) of Proposition 4.1.

Lemma 4.2. Assume $2 \alpha-1 \leq p<\infty$. Then $g$ defined in (3.1) satisfies (iv) of Proposition 4.1 with $\tau=\frac{p+1-\alpha}{\alpha}$.

Proof. A direct calculation yields that

$$
\begin{aligned}
s g^{\prime}(s)-\tau g(s)= & (p+1-\alpha) s f^{p-1}\left(f^{\prime}\right)^{2}+(\alpha-1) s f^{p-1}\left(f^{\prime}\right)^{4} \\
& +(\alpha-2) \lambda s\left(f^{\prime}\right)^{2}-(\alpha-1) \lambda s\left(f^{\prime}\right)^{4}-\tau f^{p} f^{\prime}+\tau \lambda f f^{\prime} \\
\geq & (p+1-\alpha-\alpha \tau) s f^{p-1}\left(f^{\prime}\right)^{2}+(\tau-1) \lambda s\left(f^{\prime}\right)^{2}+(\alpha-1) s f^{p-1}\left(f^{\prime}\right)^{4} .
\end{aligned}
$$

Here we used $0<f^{\prime} \leq 1$ and Lemma 2.2. Choosing $\tau=\frac{p+1-\alpha}{\alpha}$, we obtain $\tau \geq 1$ and $s g^{\prime}-\tau g>0$ for $s>0, s \neq b$. 
Lemma 4.3. Suppose $\alpha>2,2 \alpha-1 \leq p<3 \alpha-3$ and let $\tau=\frac{p+1-\alpha}{\alpha}$. Assume further

$$
\begin{aligned}
& \left(f^{\prime}(b)\right)^{2} \leq \frac{(2 \tau+2 \alpha-3)(\alpha-2)+\tau^{2}}{(\alpha-1)(2 p+3 \alpha-6)} \\
& \frac{f(b)}{b f^{\prime}(b)} \geq \frac{(p-2 \alpha+1) \alpha}{p+1-\alpha} \\
& 3 p-6 \alpha+5+4(\alpha-1)\left(f^{\prime}(b)\right)^{2}-(2 \tau-1) \frac{f(b)}{b f^{\prime}(b)} \leq 0
\end{aligned}
$$

where $b=f^{-1}\left(\lambda^{\frac{1}{p-1}}\right)$. Then $g$ satisfies (v) of Proposition 4.1.

Proof. By a direct computation, it follows

$$
\left(s\left(\frac{g}{s^{\tau}}\right)^{\prime}\right)^{\prime}=\frac{1}{s^{\tau+1}}\left(s^{2} g^{\prime \prime}+(1-2 \tau) s g^{\prime}+\tau^{2} g\right) .
$$

We claim that $s^{2} g^{\prime \prime}+(1-2 \tau) s g^{\prime}+\tau^{2} g<0$ for $s>b$. Indeed from (3.1), we have

$$
\begin{aligned}
s^{2} g^{\prime \prime}+ & (1-2 \tau) s g^{\prime}+\tau^{2} g \\
= & f^{p-2}\left(s^{2} f^{2} f^{\prime \prime \prime}+3 p s^{2} f f^{\prime} f^{\prime \prime}+(1-2 \tau) s f^{2} f^{\prime \prime}\right. \\
& \left.\quad+p(1-2 \tau) s f\left(f^{\prime}\right)^{2}+\tau^{2} f^{2} f^{\prime}+p(p-1) s^{2}\left(f^{\prime}\right)^{3}\right) \\
& \quad-\lambda\left(s^{2} f f^{\prime \prime \prime}+3 s^{2} f^{\prime} f^{\prime \prime}+(1-2 \tau) s f f^{\prime \prime}+(1-2 \tau) s\left(f^{\prime}\right)^{2}+\tau^{2} f f^{\prime}\right) \\
= & : f^{p-2} K_{1}(s)-\lambda K_{2}(s) .
\end{aligned}
$$

We prove $K_{1}(s)<0$ and $K_{2}(s)>0$ for $s>b$.

Firstly we estimate $f K_{2}(s)$. From (2.3) and (2.4), we have

$$
\begin{aligned}
f K_{2}(s)= & s^{2} f^{2} f^{\prime \prime \prime}+3 s^{2} f f^{\prime} f^{\prime \prime}+(1-2 \tau) s f^{2} f^{\prime \prime}+(1-2 \tau) s f\left(f^{\prime}\right)^{2}+\tau^{2} f^{2} f^{\prime} \\
= & 4(\alpha-1)^{2} s^{2}\left(f^{\prime}\right)^{7}-2(3 \alpha-4)(\alpha-1) s^{2}\left(f^{\prime}\right)^{5}-(2 \tau-1)(\alpha-1) s f\left(f^{\prime}\right)^{4} \\
& +2(\alpha-1)(\alpha-2) s^{2}\left(f^{\prime}\right)^{3}+(2 \tau-1)(\alpha-2) s f\left(f^{\prime}\right)^{2}+\tau^{2} f^{2} f^{\prime} .
\end{aligned}
$$

By (ii) of Lemma 2.2 and $\alpha>2$, it follows

$$
\begin{aligned}
& 2(\alpha-1)(\alpha-2) s^{2}\left(f^{\prime}\right)^{3}+(2 \tau-1)(\alpha-2) s f\left(f^{\prime}\right)^{2}+\tau^{2} f^{2} f^{\prime} \\
& \quad \geq 2(\alpha-1)(\alpha-2) s^{2}\left(f^{\prime}\right)^{3}+(2 \tau-1)(\alpha-2) s^{2}\left(f^{\prime}\right)^{3}+\tau^{2} s^{2}\left(f^{\prime}\right)^{3} \\
& \quad=\left((2 \tau+2 \alpha-3)(\alpha-2)+\tau^{2}\right) s^{2}\left(f^{\prime}\right)^{3} .
\end{aligned}
$$

By (ii) of Lemma 2.2, we also have

$$
-2(3 \alpha-4)(\alpha-1) s^{2}\left(f^{\prime}\right)^{5}-(2 \tau-1)(\alpha-1) s f\left(f^{\prime}\right)^{4} \geq-(\alpha-1)(2 p+3 \alpha-6) s^{2}\left(f^{\prime}\right)^{5} .
$$


From (4.5)-(4.7), we obtain

$f K_{2}(s) \geq 4(\alpha-1)^{2} s^{2}\left(f^{\prime}\right)^{7}-(\alpha-1)(2 p+3 \alpha-6) s^{2}\left(f^{\prime}\right)^{5}+\left((2 \tau+2 \alpha-3)(\alpha-2)+\tau^{2}\right) s^{2}\left(f^{\prime}\right)^{3}$.

From (4.1) and the fact that $\left(\left(f^{\prime}\right)^{2}\right)^{\prime}=2 f^{\prime} f^{\prime \prime}<0$, it follows

$$
\left((2 \tau+2 \alpha-3)(\alpha-2)+\tau^{2}\right)-(\alpha-1)(2 p+3 \alpha-6)\left(f^{\prime}(s)\right)^{2} \geq 0 \quad \text { for } s>b .
$$

This implies $f K_{2}(s)>0$. Since $f(s)>0$ for $s>0$, we obtain $K_{2}(s)>0$ for $s>b$.

Next we estimate $K_{1}(s)$. From (2.3) and (2.4), we have

$$
\begin{aligned}
K_{1}(s)= & s^{2} f^{2} f^{\prime \prime \prime}+3 p s^{2} f f^{\prime} f^{\prime \prime}+(1-2 \tau) s f^{2} f^{\prime \prime}+p(1-2 \tau) s f\left(f^{\prime}\right)^{2} \\
& +\tau^{2} f^{2} f^{\prime}+p(p-1) s^{2}\left(f^{\prime}\right)^{3} \\
= & 4(\alpha-1)^{2} s^{2}\left(f^{\prime}\right)^{7}+(\alpha-1)(3 p-6 \alpha+5) s^{2}\left(f^{\prime}\right)^{5} \\
& -(2 \tau-1)(\alpha-1) s f\left(f^{\prime}\right)^{4}+(p+1-\alpha)(p+1-2 \alpha) s^{2}\left(f^{\prime}\right)^{3} \\
& -(2 \tau-1)(p+1-\alpha) s f\left(f^{\prime}\right)^{2}+\tau^{2} f^{2} f^{\prime} .
\end{aligned}
$$

We claim that

$$
\begin{aligned}
& (p+1-\alpha)(p+1-2 \alpha) s^{2}\left(f^{\prime}\right)^{3}-(2 \tau-1)(p+1-\alpha) s f\left(f^{\prime}\right)^{2}+\tau^{2} f^{2} f^{\prime} \\
& \quad=s^{2}\left(f^{\prime}\right)^{3}\left((p+1-\alpha)(p+1-2 \alpha)-(2 \tau-1)(p+1-\alpha) \frac{f}{s f^{\prime}}+\tau^{2}\left(\frac{f}{s f^{\prime}}\right)^{2}\right) \\
& \quad \leq 0 \text { for } s>b .
\end{aligned}
$$

Indeed we put $\phi(s)=\frac{f(s)}{s f^{\prime}(s)}$. Then by (ii) and (iii) of Lemma 2.2, $\phi(s)$ is increasing and $1 \leq \phi(s) \leq \alpha$ for all $s>0$. We also put $p_{1}:=p+1-\alpha$. Then $\tau=\frac{p_{1}}{\alpha}$ and

$$
\begin{aligned}
(p+1-\alpha)(p+1-2 \alpha)-(2 \tau-1) & (p+1-\alpha) \frac{f}{s f^{\prime}}+\tau^{2}\left(\frac{f}{s f^{\prime}}\right)^{2} \\
= & p_{1}\left(p_{1}-\alpha\right)-\left(\frac{2 p_{1}}{\alpha}-1\right) p_{1} \phi+\frac{p_{1}^{2}}{\alpha^{2}} \phi^{2} .
\end{aligned}
$$

Finally we define for $1 \leq t \leq \alpha$

$$
\begin{aligned}
K_{3}(t) & :=p_{1} t^{2}-\left(2 p_{1}-\alpha\right) \alpha t+\alpha^{2}\left(p_{1}-\alpha\right) \\
& =(t-\alpha)\left(p_{1} t-\alpha\left(p_{1}-\alpha\right)\right) .
\end{aligned}
$$

Then it follows

$$
K_{3}(t) \leq 0 \text { for } t \in\left[\frac{\alpha\left(p_{1}-\alpha\right)}{p_{1}}, \alpha\right]
$$


Now (4.2) implies

$$
\frac{f(b)}{b f^{\prime}(b)} \geq \frac{(p-2 \alpha+1) \alpha}{p+1-\alpha}=\frac{\alpha\left(p_{1}-\alpha\right)}{p_{1}}
$$

Thus from (4.2), we have $K_{3}(t) \leq 0$ for $t \in\left[\frac{f(b)}{b f^{\prime}(b)}, \alpha\right]$. Since $\phi(s)$ is increasing, we obtain

$$
p_{1}\left(p_{1}-\alpha\right)-\left(\frac{2 p_{1}}{\alpha}-1\right) p_{1} \phi(s)+\frac{p_{1}^{2}}{\alpha^{2}} \phi^{2}(s) \leq 0 \quad \text { for } s>b
$$

and hence (4.9) holds.

Next we observe that

$$
\begin{aligned}
& 4(\alpha-1)^{2} s^{2}\left(f^{\prime}\right)^{7}+(\alpha-1)(3 p-6 \alpha+5) s^{2}\left(f^{\prime}\right)^{5}-(2 \tau-1)(\alpha-1) s f\left(f^{\prime}\right)^{4} \\
& =(\alpha-1) s^{2}\left(f^{\prime}\right)^{5}\left(3 p-6 \alpha+5-(2 \tau-1)\left(\frac{f}{s f^{\prime}}\right)+4(\alpha-1)\left(f^{\prime}\right)^{2}\right) \\
& =:(\alpha-1) s^{2}\left(f^{\prime}\right)^{5} K_{4}(s) .
\end{aligned}
$$

We can easily see that $K_{4}^{\prime}(s)=-(2 \tau-1)\left(\frac{f}{s f^{\prime}}\right)^{\prime}+8(\alpha-1) f^{\prime} f^{\prime \prime}<0$, that is, $K_{4}(s)$ is decreasing. Moreover from (4.3), we have

$$
K_{4}(b)=3 p-6 \alpha+5-(2 \tau-1)\left(\frac{f(b)}{b f^{\prime}(b)}\right)+4(\alpha-1)\left(f^{\prime}(b)\right)^{2} \leq 0
$$

Thus we obtain $K_{4}(s) \leq 0$ for $s>b$. From (4.8)-(4.10), it follows $K_{1}(s) \leq 0$ for $s>b$. Thus from (4.4), we obtain $\left(s\left(\frac{g}{s^{\tau}}\right)^{\prime}\right)^{\prime}<0$ for $s>b$.

Lemma 4.4. Suppose $\alpha>2,2 \alpha-1 \leq p<3 \alpha-3$ and let $\tau=\frac{p+1-\alpha}{\alpha}$. Then

(i) For every fixed $\kappa>0$, there exists $\lambda_{0}>0$ such that if $\lambda \geq \lambda_{0}$, (4.1)-(4.3) in Lemma 4.3 are fulfilled.

(ii) For every fixed $\lambda>0$, there exists $\kappa_{0}>0$ such that if $\kappa \geq \kappa_{0}$, (4.1)-(4.3) in Lemma 4.3 are fulfilled.

Proof. From (2.2) and $b=f^{-1}\left(\lambda^{\frac{1}{p-1}}\right)$, it follows

$$
\left(f^{\prime}(b)\right)^{2}=\frac{1}{1+\alpha \kappa \lambda^{\frac{2 \alpha-2}{p-1}}} .
$$

Since $\frac{(2 \tau+2 \alpha-3)(\alpha-2)+\tau^{2}}{(\alpha-1)(2 p+3 \alpha-6)}>0,(4.1)$ is satisfied if either $\lambda$ or $\kappa$ is sufficiently large. 
Next we observe that

$$
b=f^{-1}\left(\lambda^{\frac{1}{p-1}}\right)=\int_{0}^{\lambda^{\frac{1}{p-1}}} \sqrt{1+\alpha \kappa t^{2 \alpha-2}} d t .
$$

This implies $b \rightarrow \infty$ if either $\lambda$ or $\kappa$ tends to infinity. By (iii) of Lemma 2.3 and the fact

$$
\frac{(p-2 \alpha+1) \alpha}{(p+1-\alpha)}<\alpha
$$

(4.2) holds if either $\lambda$ or $\kappa$ is sufficiently large.

Finally we notice that if $p<3 \alpha-3$, then

$$
\frac{3 p-6 \alpha+5}{2 \tau-1}<\alpha
$$

Since $\frac{f(b)}{b f^{\prime}(b)} \nearrow \alpha$ and $f^{\prime}(b) \rightarrow 0$ as $b \rightarrow \infty$, (4.3) holds if $b$ is sufficiently large. Since $b \rightarrow \infty$ as either $\lambda$ or $\kappa$ goes to infinity, (4.3) is fulfilled if either $\lambda$ or $\kappa$ is sufficiently large.

By Lemmas 4.2-4.4, we can apply Proposition 4.1. Hence we obtain the uniqueness of positive radial solutions of (1.2) for $N=2$ and the proof of Theorem 1.3 is complete.

\section{Non-degeneracy of the ground state solution}

As we have shown in Sections 3 and 4, problem (1.2) and (2.5) has at most one positive radial solution. In this section, we study spectral properties of the linearized operator. We will show that the unique positive radial solution of $(2.5)$ is non-degenerate in $H_{\text {rad }}^{1}\left(\mathbb{R}^{N}\right)$ if we add some conditions. To this aim, we apply the result due to Bates and Shi $[\mathbf{2}]$. We consider the following semilinear problem:

$$
-\Delta u=g(u) \text { in } \mathbb{R}^{N}, u>0, u(x)=u(|x|), u \rightarrow 0 \text { as }|x| \rightarrow \infty, u(0)=\max u(x) .
$$

Proposition $5.1[2]$. Suppose $g \in C^{1}([0, \infty))$ satisfies the following properties.

(g1) There exists $b>0$ such that $g(0)=g(b)=0, g(s)<0$ for $s \in(0, b), g^{\prime}(0)<0$ and $g^{\prime}(b)>0$.

(g2) There exists $\theta>b$ such that $\int_{0}^{\theta} g(s) d s=0$.

(g3) $g(s)>0$ for all $s>b$.

(g4) $K_{g}(s)$ (defined in $\left.(3.1)\right)$ is non-increasing in $[\theta, \infty)$ and $K_{g}(s) \rightarrow K_{\infty} \in\left[1, \frac{N+2}{N-2}\right)$ as $s \rightarrow \infty$. 
(g5) $K_{g}(s) \geq K_{g}(\theta)$ for $s \in(b, \theta]$ and $K_{g}(s) \leq K_{\infty}$ for $s \in(0, b)$.

Under assumptions (g1)-(g5), let $u$ be the unique positive radial solution of (5.1) and $L_{0}:=-\Delta-g^{\prime}(0)$. Then

(1) $\sigma\left(L_{0}\right)=\sigma_{p}\left(L_{0}\right) \cup \sigma_{e}\left(L_{0}\right)$.

(2) $\sigma_{e}\left(L_{0}\right)=\left[-g^{\prime}(0), \infty\right), \sigma_{p}\left(L_{0}\right) \subset\left(-\infty,-g^{\prime}(0)\right)$.

(3) If $\mu \in \sigma_{p}\left(L_{0}\right)$, then the corresponding eigenfunction $\phi(x)$ satisfies

$$
|\phi(x)| \leq C_{\epsilon} e^{-\sqrt{\frac{-g^{\prime}(0)-\mu+\epsilon}{2}}|x|} \quad \text { for } x \in \mathbb{R}^{N}
$$

for any small $\epsilon>0$ and some $C_{\epsilon}>0$.

(4) If $\mu \in \sigma_{p}\left(L_{0}\right) \cap(-\infty, 0)$, then the corresponding eigenfunction is radially symmetric.

(5) The principal eigenvalue $\mu_{1}\left(L_{0}\right)<0$ is simple and the corresponding eigenfunction $\phi_{1}$ can be chosen to be positive.

(6) $\mu_{2}\left(L_{0}\right)=0$ and the eigenspace corresponding to the eigenvalue $\mu=0$ is spanned by

$$
\left\{\frac{\partial u}{\partial x_{i}} ; i=1, \cdots, N\right\}
$$

We show that (g1)-(g5) hold for $g(s)$ defined in (3.1). We can easily see that (g1) and (g3) hold. Next we put $\theta=\tilde{f}\left(\left(\frac{p+1}{2} \lambda\right)^{\frac{1}{p-1}}\right)$. Then we have $\int_{0}^{\theta} g(s) d s=0$, which implies (g2). Next we show that if we suppose additional conditions on $\alpha$ and $p$, then (g4) and (g5) hold.

Lemma 5.2. Assume $2 \alpha-1 \leq p<\frac{(2 \alpha-1) N+2}{N-2}$. Then $g(s)$ defined in (3.1) satisfies

$$
\lim _{s \rightarrow \infty} K_{g}(s)=\frac{p+1-\alpha}{\alpha}=: K_{\infty} \in\left[1, \frac{N+2}{N-2}\right)
$$

Proof. By direct computation, we have

$$
\begin{aligned}
K_{g}(s) & =\frac{g^{\prime}(s) s}{g(s)} \\
& =\frac{(p-1) f^{p-1}\left(f^{\prime}\right)^{2} s+(2-\alpha)\left(f^{\prime}\right)^{2} s\left(f^{p-1}-\lambda\right)+(\alpha-1)\left(f^{\prime}\right)^{4} s\left(f^{p-1}-\lambda\right)}{f f^{\prime}\left(f^{p-1}-\lambda\right)} \\
& =\frac{s f^{\prime}}{f}\left(\frac{(p-1) f^{p-1}}{f^{p-1}-\lambda}+(2-\alpha)+(\alpha-1)\left(f^{\prime}\right)^{3}\right) .
\end{aligned}
$$


Since $\lim _{s \rightarrow \infty} \frac{s f^{\prime}}{f}=\frac{1}{\alpha}$ and $\lim _{s \rightarrow \infty} f^{\prime}=0$, we obtain

$$
\lim _{s \rightarrow \infty} K_{g}(s)=\frac{p-1}{\alpha}+\frac{2-\alpha}{\alpha}=\frac{p+1-\alpha}{\alpha} .
$$

From $2 \alpha-1 \leq p<\frac{(2 \alpha-1) N+2}{N-2}$, it follows $\frac{p+1-\alpha}{\alpha} \in\left[1, \frac{N+2}{N-2}\right)$. Thus we show the claim of Lemma 5.2.

Lemma 5.3. Assume $\alpha>2,2 \alpha-1 \leq p<3 \alpha-3$ and

$$
\frac{p}{\alpha(3 \alpha-p-3)} \leq \kappa \lambda^{\frac{2 \alpha-2}{p-1}}
$$

Then $g(s)$ defined in (3.1) satisfies (g4) and (g5) in Proposition 5.1.

Proof. Firstly we observe that if $\alpha>2$ and $2 \alpha-1 \leq p<3 \alpha-3$, then

$$
\frac{\alpha-p}{\alpha(p-1)}<\frac{p}{\alpha(3 \alpha-p-3)} \text {. }
$$

In fact, we have

$$
(\alpha-p)(3 \alpha-p-3)-p(p-1)=(3 \alpha-4 p)(\alpha-1) .
$$

Since $\alpha>2$ and $p \geq 2 \alpha-1$, it follows

$$
3 \alpha-4 p \leq-5 \alpha+4<-6<0 .
$$

This implies $(3 \alpha-4 p)(\alpha-1)<0$ and hence (5.2) holds.

By Lemmas 3.2 and 5.2, we can see (g4) and the first inequality in (g5) hold. Next we show that $K_{g}(s) \leq 1$ for $s \in(0, b)$. In fact, we have

$$
\begin{aligned}
s g^{\prime}(s)-g(s)= & (p-1) f^{p-1}\left(f^{\prime}\right)^{2} s+(2-\alpha)\left(f^{\prime}\right)^{2} s\left(f^{p-1}-\lambda\right) \\
& +(\alpha-1)\left(f^{\prime}\right)^{4} s\left(f^{p-1}-\lambda\right)-f f^{\prime}\left(f^{p-1}-\lambda\right) \\
= & (p-1) f^{p-1}\left(f^{\prime}\right)^{2} s+\left(f^{p-1}-\lambda\right)\left((2-\alpha)\left(f^{\prime}\right)^{2} s+(\alpha-1)\left(f^{\prime}\right)^{4} s-f f^{\prime}\right) .
\end{aligned}
$$

For $s \in(0, b)$, it follows $f^{p-1}-\lambda<0$. On the other hand by Lemma 2.2, we have $\left(f^{\prime}\right)^{2} s \leq f f^{\prime}$. Using $f^{\prime} \leq 1$, we obtain

$$
\begin{aligned}
(2-\alpha)\left(f^{\prime}\right)^{2} s+(\alpha-1)\left(f^{\prime}\right)^{4} s-f f^{\prime} & \leq(2-\alpha)\left(f^{\prime}\right)^{2} s+(\alpha-1)\left(f^{\prime}\right)^{4} s-\left(f^{\prime}\right)^{2} s \\
& =(\alpha-1)\left(f^{\prime}\right)^{2} s\left(\left(f^{\prime}\right)^{2}-1\right) \leq 0 .
\end{aligned}
$$

Thus we have $s g^{\prime}(s)-g(s) \geq(p-1) f^{p-1}\left(f^{\prime}\right)^{2} s>0$ for all $s \in(0, b)$. Since $g(s)<0$ for $s \in(0, b)$, it follows $K_{g}(s) \leq 1$ for $s \in(0, b)$.

Finally by Lemma 5.2 , we have

$$
K_{g}(s) \leq 1 \leq K_{\infty}=\frac{p+1-\alpha}{\alpha} .
$$

Thus the second inequality in (g5) holds.

By Lemmas 5.2 and 5.3, we obtain the following 
Theorem 5.4. Assume $N \geq 3, \alpha>2,2 \alpha-1 \leq p<3 \alpha-3$ and

$$
\frac{p}{\alpha(3 \alpha-p-3)} \leq \kappa \lambda^{\frac{2 \alpha-2}{p-1}}
$$

Let $v$ be the unique positive solution of $(2.5)$ and $L:=-\Delta-g^{\prime}(v) ; H^{2}\left(\mathbb{R}^{N}\right) \rightarrow L^{2}\left(\mathbb{R}^{N}\right)$. Then

(1) $\sigma(L)=\sigma_{p}(L) \cup \sigma_{e}(L)$.

(2) $\sigma_{e}(L)=[\lambda, \infty), \sigma_{p}(L) \subset(-\infty, \lambda)$.

(3) If $\mu \in \sigma_{p}(L)$, then the corresponding eigenfunction $\phi(x)$ satisfies

$$
|\phi(x)| \leq C_{\epsilon} e^{-\sqrt{\frac{\lambda-\mu+\epsilon}{2}}|x|} \quad \text { for } x \in \mathbb{R}^{N}
$$

for any small $\epsilon>0$ and some $C_{\epsilon}>0$.

(4) If $\mu \in \sigma_{p}(L) \cap(-\infty, 0)$, then the corresponding eigenfunction is radially symmetric.

(5) The principal eigenvalue $\mu_{1}(L)<0$ is simple and the corresponding eigenfunction $\phi_{1}$ can be chosen to be positive.

(6) $\mu_{2}(L)=0$ and the eigenspace corresponding to the eigenvalue $\mu=0$ is spanned by

$$
\left\{\frac{\partial v}{\partial x_{i}} ; i=1, \cdots, N\right\}
$$

From (6) of Theorem 5.4, we obtain the non-degeneracy of the ground state solution of $(2.5)$ in $H_{r a d}^{1}\left(\mathbb{R}^{N}\right)$.

Corollary 5.5. Let $v$ be the unique positive solution of (2.5). Then $v$ is non-degenerate in $H_{r a d}^{1}\left(\mathbb{R}^{N}\right)$, that is, if

$$
-\Delta \phi-g^{\prime}(v) \phi=0 \text { in } \mathbb{R}^{N} \text { and } \phi \in H_{r a d}^{1}\left(\mathbb{R}^{N}\right),
$$

then $\phi \equiv 0$.

Remark 5.6. We have shown the non-degeneracy of the ground state solution of semilinear problem (2.5). However we don't know this implies the non-degeneracy for the ground state solution of quasilinear problem (1.2).

\section{References}

[1] S. Adachi, T. Watanabe, $G$-invariant positive solutions for a quasilinear Schrödinger equation, preprint. 
[2] P. Bates, J. Shi, Existence and instability of spike layer solutions to singular perturbation problems, J. Funct. Anal. 196 (2002), 429-482.

[3] H. Berestycki, T. Gallouët, O. Kavian, Équations de Champs scalaires euclidiens non linéaires dans le plan, C. R. Acad. Sci. Paris Sér. I Math. 297 (1983), 307-310.

[4] H. Berestycki, P. L. Lions, Nonlinear scalar fields equations, I. Existence of a ground state, Arch. Rational Mech. Anal. 82 (1983), 313-345.

[5] L. Brizhik, A. Eremko, B. Piette, W. J. Zakrzewski, Electron self-trapping in a discrete two-dimensional lattice, Physica D 159 (2001), 71-90.

[6] J. Byeon, L. Jeanjean, M. Maris, Symmetry and monotonicity of least energy solutions, Calc. Var. PDE 36 (2009), 481-492.

[7] M. Colin, L. Jeanjean, Solutions for a quasilinear Schrödinger equation: a dual approach, Nonlinear Anal. TMA. 56 (2004), 213-226.

[8] M. Colin, L. Jeanjean, M. Squassina, Stability and instability results for standing waves of quasi-linear Schrödinger equations, preprint.

[9] C. Cortázar, M. Elgueta, P. Felmer, Uniqueness of positive solutions of $\Delta u+f(u)=0$ in $\mathbb{R}^{N}, N \geq 3$, Arch. Rat. Mech. Anal. 142 (1998), 127-141.

[10] B. Gidas, W. M. Ni, L. Nirenberg, Symmetry of positive solutions of nonlinear elliptic equations in $\mathbb{R}^{N}$, Adv. Math. Studies A 7 (1981), 369-402.

[11] J. Hirata, N. Ikoma, K. Tanaka, Nonlinear scalar field equations in $\mathbb{R}^{N}$ : mountain pass and symmetric mountain pass approaches, preprint.

[12] M. Kwong, L. Zhang, Uniqueness of the positive solution of $\Delta u+f(u)=0$ in an annulus, Diff. Int. Eqns. 4 (1991), 583-599.

[13] S. Kurihara, Large-amplitude quasi-solitons in superfluid films, J. Phys. Soc. Japan 50 (1981), 3262-3267.

[14] J.-Q. Liu, Z.-Q. Wang, Soliton solutions for quasilinear Schrödinger equations, Proc. Amer. Math. Soc. 131 (2003), 441-448.

[15] J.-Q. Liu, Y.-Q. Wang, Z.-Q. Wang, Soliton solutions for quasilinear Schrödinger equations, II, J. Diff. Eqns. 187 (2003), 473-493.

[16] J.-Q. Liu, Y.-Q. Wang, Z.-Q. Wang, Solutions for quasi-linear Schrödinger equations via the Nehari method, Comm. PDE 29 (2004), 879-901.

[17] K. Mcleod, J. Serrin, Uniqueness of positive radial solutions of $\Delta u+f(u)=0$ in $\mathbb{R}^{N}$, Arch. Rat. Mech. Anal. 99 (1987), 115-145.

[18] M. Poppenberg, K. Schmitt, Z.-Q. Wang, On the existence of soliton solutions to quasilinear Schrödinger equations, Calc. Var. PDE 14 (2002), 329-344.

[19] J. Serrin, M. Tang, Uniqueness of ground states for quasilinear elliptic equations, Indiana Univ. Math. J. 49 (2000), 897-923. 